\title{
3-S35-1 Symposium35
}

\section{Requirement for neuropeptide $\mathrm{Y}$ in the development of allergen-induced airway hyperresponsiveness and inflammation}

\section{Nobuaki Miyahara}

Dept. Med., Technology., Okayama Univ. Graduate School

Neuropeptide Y (NPY) is a neurotransmitter that is widely expressed in the brain and peripheral nervous system. Various immune cells express the NPY Y1 receptor. NPY modulates these cells via its Y1 receptor; however, involvement of NPY in the pathophysiology of bronchial asthma, particularly airway hyperresponsiveness (AHR), has not been defined. NPY-deficient and wild-type mice were intranasally sensitized and challenged to house dust mite (HDM) extract, and airway responses were monitored. After sensitization and challenge, NPY-deficient mice showed significantly lower AHR than wild-type mice, and numbers of eosinophils and levels of type-2 cytokines [interleukin (IL)-4, IL-5, and IL-13] in bronchoalveolar lavage fluid were significantly lower. Type-2 cytokine production from splenic mononuclear cells of HDM-sensitized mice was also significantly lower in NPY-deficient mice. Treatment with a NPY receptor antagonist, significantly suppressed development of HDM-induced AHR and inflammation in wild-type mice. These data identify an important contribution of NPY to allergen-induced AHR and inflammation. Thus, manipulating NPY represents a novel therapeutic target to control allergic airway responses. 